\title{
Tan fuerte como su eslabón más débil. El caso de las AMPA en la integración escolar y social de las familias inmigradas
}

\section{As strong as its weakest link. The case of PAs in the social and school inclusion of migrant families}

\author{
Alejandro PANIAGUA RODRIGUEZ \\ Grupo EMIGRA - CER Migraciones - Universidad Autónoma de Barcelona
}

Recibido: Marzo 2012

Aceptado: Junio 2012

\section{Resumen}

Este artículo presenta una síntesis de los resultados del proyecto de investigación llevada a cabo en el curso 2009-2010 en la Federación de Asociaciones de Padres y Madres de Alumnos de Cataluña $(\mathrm{FaPaC})$. A partir de la identificación del espacio de las AMPA como un caso específico y privilegiado en las relaciones familia-escuela, los objetivos de la investigación se han centrado en dos ejes. En primer lugar, plantear y evaluar el posible papel de las AMPA como una verdadera asociación intermediaria donde construir proyectos y como recurso socioeducativo. En segundo lugar, para analizar y valorar el grado de articulación entre la problemática social detectada - el déficit de participación de las familias inmigradas como explicación a los peores resultados escolares-, las AMPA y las intervenciones y proyectos institucionales que, según el contexto específico catalán con relación a las últimas tendencias en política educativa, cabría esperar.

Palabras clave: Participación, familias inmigradas, asociaciones de padres y madres, organizaciones intermediarias, racismo institucional, capital social, política educativa.

\begin{abstract}
This paper presents a summary of the results of a Project carried out by the author on behalf of the Catalan Federation of Parents' Associations ( $\mathrm{FaPaC})$, where we identify PAs as a specific and privileged case of family-school relations. The Project intended to assess the potential role Pas could play as intermediary organizations enhancing social integration and analyze the degree of the relation between the social problem constructed - migrant families' lack of participation as an explanation for the lower school performance of their children - and the institutional projects that should be expected in the Catalan context among PAs.
\end{abstract}

Keywords: Participation, immigrant families, Parents' Associations, Intermediary Organizations, Institutional Racism, Social Capital, Educational Policy. 
La necesidad de definir estrategias óptimas y de resituar el papel de la comunidad educativa y los agentes sociales para la mejora de los resultados académicos fue una de las prioridades del gobierno progresista catalán en su primera legislatura ${ }^{1}$. Así, los principios y filosofía que enmarcaron el Pla de Llengua i Cohesió Social (2004) y el de los Plans Educatius d'Entorn (2005) supusieron ingentes recursos económicos y humanos para el desarrollo de esta perspectiva sociofamiliar.

Una perspectiva y un discurso institucional que no ha estado muy alejado del sentido común de muchos docentes. $Y$ es que la dejadez, permisividad o el bajo interés y participación atribuidas a muchas familias - especialmente si éstas son inmigradas o de clases populares - ha cristalizado como parte del bricolage del educador en forma de trinomio entre baja participación, que equivale a desinterés y el cual desemboca en la explicación del fracaso o las dificultades en la escuela de muchos alumnos.

Hablar del papel de las familias en los resultados escolares nos remite a dos problemáticas bien distintas que suelen confundirse. Y peor aún, arrincona a una tercera problemática que corre el riesgo de pasar desapercibida cuando hablamos de éxito escolar. Pese a todo, como veremos, las tres están íntimamente relacionadas.

En primer lugar, es central separar el quién del cómo, es decir, qué familias y cómo actúan éstas en relación a los procesos de escolarización de sus hijos e hijas. O si se prefiere, separar la influencia de la familia per se de la participación per se. Así, esta separación nos permite identificar, por ejemplo, que muchas familias poseen una serie de capitales que les confiere una ventaja por cuanto éstos están armonizadas con la cultura escolar (de Carvalho, 2001; Stanton-Salazar, 1997; Lareau, 1987).

La familia y su contexto socioeconómico puede afectar y de hecho afecta estructuralmente y sin necesidad alguna de participación la realidad escolar de muchos alumnos (Tapia, 2002), cuyos progenitores, por ejemplo, distan mucho de poder acceder a los circuitos diferenciados y privilegiados de escolarización (Brown, 1990) o de trabajar el sistema a su favor (Lareau \& Horvat, 1999).

En segundo lugar, centrándonos ahora en el cómo de las familias, nos encontramos ante una lista muy amplia de modelos conceptuales de intervención: desde los 6 modelos de Epstein (2002), pasando por muy diversos modelos psicologistas, ecológicos y procesuales (resumidos en Corter and Pelletier, 2005), recogiendo diversos ámbitos - centrado en los padres; centrado en la escuela; centrado en el hogar -, que otras síntesis sintetizan de manera genérica entre dos pares de opuestos: la intervención directa en el desarrollo de habilidades escolares o la implicación diferida dirigida a motivar y apoyar emocionalmente (Pomerantz et al., 2007; Desforges \& Abouchaar, 2003). Lo cual evita cualquier aproximación simplista al concepto de participación y da cuenta de la complejidad a que cualquier intervención sociofamiliar debe hacer frente.

${ }^{1}$ La coalición progresista formada por PSC, ERC e ICV-EUiA que ha gobernado durante dos legislaturas, entre el 2003-2010, tras más de 20 años de hegemonía de la derecha en Cataluña. 
En tercer lugar, este énfasis en la participación suele dirigirse hacia unos colectivos específicos, construidos de manera deficitaria por la escuela (Crozier \& Davies, 2007; Theodorou, 2007; Crozier, 2001), manteniendo las prácticas escolares internas ocultas y ajenas de toda culpa o responsabilidad (Dahlstedt, 2009). De manera que los procesos de jerarquización y estratificación escolar interna continúan condicionando la experiencia de aprendizaje y de socialización del alumnado (Carrasco et al., 2010), y de esos resultados académicos que se quieren mejorar a partir de la implicación familiar.

En relación a la literatura existente sobre la relación entre familia, participación y éxito escolar, ésta se muestra contradictoria y no concluyente, donde subyace el aparente divorcio entre el hecho que la familia es la variable que mejor predice y explica los procesos y resultados escolares (López et al., 2005; Desforges \& Abouchaar, 2003; Boethel, 2003) y el irregular y ambiguo impacto de los proyectos y políticas educativas basadas en la implicación y participación de los padres (Pomerantz et al., 2007; Corter \& Pelletier, 2005; Driessen et al., 2004; Desforges \& Abouchaar, 2003).

Parte del problema que de forma reiterada señalan los autores críticos o cautos con esta conexión entre participación y mejora del éxito escolar es la dificultad para esclarecer, entre la amalgama de factores que inciden en el éxito académico, aquéllos que, de manera específica, remiten al papel de los padres. Por ejemplo, Corter \& Pelletier (2005) señalan como la mayoría de estudios se basan en escritos en defensa de tales procesos per se o son estudios poco rigurosos, muy locales y anecdóticos. Por otro lado, Theodorou (2007) pasa revista a las asunciones sobre la participación de los padres y las problematiza para desenmascarar su naturaleza culturalmente específica y sus consecuencias negativas para la inclusión de los grupos más vulnerables. Symeou (2005), en otra exhaustiva revisión, pone el énfasis en las múltiples dificultades que, para maestros y familias, supone la activación o mejora de las relaciones familia/escuela. Por otro lado, Poomerantz et al. (2007) nos alertan que la cuestión no es en que se participe, sino en el cómo y con qué objetivos, es decir, en la calidad de la participación.

Por último, encontramos el discurso de los protagonistas, esto es, de las propias familias inmigradas, cuya voz es silenciada en los discursos del personal educativo y de los responsables políticos, pese a que cuando analizamos estudios realizados con familias inmigradas (Carrasco et al., 2009; Crozier \& Davies, 2007; Abrams \& Gibbs, 2002) es coincidente el hecho de que éstas se preocupan mucho por la educación de sus hijos, intentan involucrarse en la medida que pueden y hallan grandes barreras a la hora de participar en igualdad de condiciones que los otros padres.

\section{Situando a las AMPA en la relación familia-escuela: estudios previos y propuesta de investigación.}

Según el informe de Eurydice sobre el papel de las familias en los sistemas educativos europeos (1997), el tema de la participación en la escuela surgió en la 
mayoría de países entre los años 70 y 80 , constituyéndose un corpus legal a tal efecto y creándose un conjunto de semejanzas "formales" en los tipos de órganos donde las familias están representadas, su alcance en las competencias y la presencia de las asociaciones de padres y madres, a nivel de escuela, en la totalidad de países. Sin embargo, como señala Symeou (2005), es un error pensar que las relaciones familia y la escuela han evolucionado de manea lineal y homogénea, ya que existen una multitud de variables que afectan esta relación y buena parte de ellos llevan la impronta del contexto social más amplio.

En el caso de España, por ejemplo, la implementación de una legislación formal ha ido acompañada de una caída paulatina y muy clara de la participación en los espacios que, precisamente, han sido legislados a tal efecto (Garreta, 2008a; Subirats et al. 2003), algo muy similar a lo acontecido para el caso de Dinamarca, por ejemplo (Kristoffersson, 2007).

En el Estado Español han predominado los estudios sobre la participación de las familias en los órganos formales de los centros educativos (Sarramona, 2001; Santos Guerra, 1997; Feito 1992) y los trabajos basados en encuestas y entrevistas a miembros de AMPA y el resto de la comunidad educativa donde se tratan cuestiones muy amplias y genéricas sobre su funcionamiento y sobre temas de participación y las relaciones con la escuela (Garreta, 2008a y 2008b; Pañellas \& Aguacil, 2008; Sarramona, 2001).

Un tercer caso lo componen un grupo mixto de trabajos que van desde las orientaciones prácticas aparecidas en su mayoría en publicaciones para maestros o manuales de orientación, hasta las propuestas metodológicas de investigación-acción y reflexiones psicopedagógicas sobre la importancia de la coherencia y la continuidad entre el espacio familiar y el educativo (Martínez-González \& Pérez-Herrero, 2006).

El estudio cuantitativo realizado por Garreta (2008b), nos advierte sobre la problemática incorporación de familias inmigradas a las AMPA, como estas asociaciones son muy débiles o ni siquiera existen en contextos de fuerte inmigración o la relativamente baja participación a las reuniones y actividades convocadas (18\% y $32 \%$, respectivamente), destacando las escasas actuaciones llevadas a cabo para favorecer esta participación por parte de las AMPA (27\%) y como éstas son informativas y puntuales.

Desde el marco para la participación propuesto por Epstein et al. (2002) las AMPA efectúan potencialmente sus seis tipos de implicación familiar: ofrecen cursos para mejorar la crianza de los niños y la actuación como educadores de los padres en casa; son un canal de comunicación con la escuela y actúan en las tomas de decisión a través del consejo escolar; en muchos casos colaboran con el entorno del barrio y gestionan la ayuda de los padres hacia la escuela en determinadas actividades - excursiones, fiestas, eventos culturales -.

Por otro lado, las Asociaciones de Padres se asemejan a las funciones de las organizaciones intermediarias que López et al. (2005) consideran esenciales para la construcción de capacidades para la participación de las familias: reforzar un sentido de la eficacia, construir capital cultural a través de la adquisición de conocimiento y 
habilidades prácticas; facilitar el desarrollo de las redes sociales; desarrollar un sentido de liderazgo; facilitar los lazos entre la cultura familiar y la escolar así como lubricar los canales de comunicación y de relación con la escuela a través del diálogo, el debate de temas educativos y proveyendo espacios para que maestros y distintos tipos de familias aprendan las unas de las otras.

Por consiguiente, es relevante preguntarnos qué papel pueden jugar las AMPA, como intermediarias entre el espacio escolar y el espacio familiar, en el fomento de la participación de los grupos más desfavorecidos y, en tal caso, qué impacto podría tener esta intervención con las condiciones de escolarización y las trayectorias escolares de los hijos e hijas de las familias inmigradas.

\section{Capital social, movilización de recursos y asociaciones intermediarias. Interrogantes de la investigación (I)}

La aproximación desde la teoría del capital social ha sido empleada por múltiples autores para explicar de manera más clara la desigualdad educativa a partir de una aproximación más ecológica, que nos permite explicar como el desarrollo o la mejora de recursos comunitarios no estrictamente económicos puede contribuir a contrarrestar la posición subordinada de las familias inmigradas y sus hijos e hijas en la escuela (Carrasco et al., 2009; Bolivar, 2006; Monkman et al., 2005; Torres Sánchez, 2004).

Como señala Stanton-Salazar (1997), las redes sociales actúan como vías de privilegio y poder, convirtiéndose, a la vez, en fuentes de reproducción social y formas de superar barreras institucionales si se desarrollan de una manera estratégica para trascender la base escasa y conservativa que suele caracterizar la red social de los grupos más desfavorecidos. Por otro lado, el factor étnico, en relación al proyecto migratorio de las familias inmigradas, puede funcionar como recurso educativo partir de la evidencia de las altas expectativas que estas familias depositan en la educación de sus hijos, lo cual contribuiría a reforzar el sentimiento de seguridad, la percepción del apoyo y la sensación de reconocimiento (Carrasco et al., 2009).

Central desde esta perspectiva sería la capacidad de activación normativamente válida de ciertas formas de capital social asociadas a las clases medias para trabajar el sistema (Lareu \& Horvat, 1999), es decir, la capacidad de movilizar recursos "con una orientación de clase media" (Dywer \& Moddod et al., 2006) a partir de tener información específica sobre el sistema educativo o el desarrollo de habilidades y valores requeridos para el éxito en la escuela.

$\mathrm{Al}$ analizar la capacidad de interrumpir las lógicas de exclusión educativa a partir de las reformas para mejorar la presencia de las familias más silenciadas (Abrams \& Gibbs, 2002) y el papel potencial que las organizaciones intermediarias descritas anteriormente pueden jugar a la hora de empoderar a las familias, mejorando su capacidad de intervenir y relacionarse con los profesionales así como sus conocimientos sobre lo escolar (López et al., 2005), estamos en condiciones de formular las siguientes preguntas: 
- ¿En qué aspecto los proyectos de AMPA suponen una oportunidad para mejorar la vinculación de las familias inmigradas como asociaciones intermediarias?

- ¿Pueden convertirse en un espacio real de fortalecimiento de las redes sociales de las familias? (Torres Sánchez, 2004)

\section{Neoliberalismo y racismo institucional. Interrogantes de la investigación (II)}

A pesar de la defensa de la participación como una herramienta para empoderar a las familias, otros autores han señalado que las políticas y programas para fomentar la involucración de las familias en la escuela podrían fortalecer los procesos de exclusión. La existencia de un racismo institucional (Dahlstedt, 2009; Warren, 2007; Theodorou, 2007; Crozier, 2001) que se niega a reconocer como los aspectos étnicos efectivamente hacen que las experiencias sociales y educativas de las minorías étnicas sean diferentes al del resto de la población, provoca la construcción homogénea y deficitaria de dichas familias como grupo social, a partir de una construcción del buen padre y del cómo y cuándo de la participación, que pasa por alto los intereses y las dinámicas comunitarias propias que son distintas de la cultura hegemónica proyectada desde la escuela.

Gitlin et al. (2003) nos muestran como las pautas de exclusión y segregación pueden generarse a partir de mecanismos y discursos de inclusión y aceptación de la diferencia, remarcando el papel que otras fuerzas más amplias juegan en los procesos concretos y cotidianos del espacio escolar. En este sentido, los nuevos proyectos neoliberales de la escuela, a partir de sus finalidades economicistas, la creación de los mercados educativos y penetración del management organizacional (Laval, 2005) puede minar las prácticas inclusivas comprensivas y de atención a la diversidad de los grupos étnicos (Gomolla, 2006), afianzando estrategias de evitación y procesos análogos que recrean nuevas formas de desigualdad social y étnica así como la segregación (Van Zanten, 1997). De este modo, es clave preguntarnos:

- ¿Hasta qué punto no son las AMPA un espacio demasiado formalizado, que no hace sino dificultar el acceso de ciertas familias, siguiendo la advertencia que hacen Taylor \& Percy-Smith (2008)?

\section{Diseño y metodología}

La presente investigación se ha llevado a cabo en el marco de un proyecto mayor en la federación de AMPAS de Cataluña $(\mathrm{FaPaC})$, que ha reportado inesperados marcos y fuentes de análisis que se inscriben en el campo propio de la etnografia institucional (Grahame \& Grahame, 2001).

El trabajo de investigación ha consistido en entrevistas a miembros de AMPA de escuelas con presencia notable o alta de familias inmigradas y de diferentes agentes educativos e institucionales que intervienen en ellas. Nos hemos centrado en las 
escuelas de primaria debido a una mayor existencia de AMPA en dichas escuelas y la mayor implicación e interés por parte de los padres en los asuntos educativos en esta franja de edad (Garreta, 2008a; Sarramona, 2001; Santos Guerra, 1997).

Por otro lado, es necesario esclarecer una cuestión, ¿qué figura exacta se esconde tras el AMPA? Es decir, es necesario dar cuenta de las múltiples formas en que las entrevistas se han dado: a veces con toda la junta del AMPA; a veces sólo con el Presidente o con el miembro más activo; y a veces, incluso, miembros de Junta con la directora o miembros de la dirección del centro. Esta diversidad muestra el carácter del acceso a las AMPA, como conjunto de padres y madres. Un acceso que, mayoritariamente, solo se da una vez al mes, en horas complicadas y a las cuales se necesitaría una confianza que el mismo planteamiento de la investigación imposibilita.

La selección de los centros se ha realizado a partir de la información de dinamizadoras de zona, responsables de la administración e investigadores o grupos de investigación, en relación con casos interesantes de relativo éxito a la hora de fomentar la participación de las familias en la escuela en general, y de las familias inmigradas en particular. También se ha efectuado una selección aleatoria a partir de la selección de entornos con mucha diversidad cultural.

En cualquier caso, se ha intentado que la muestra sea lo más diversificada posible ateniendo a distintas tipologías de AMPA, contexto de la población, grado de presencia de población inmigrada, relación del AMPA con el centro o existencia de proyectos de centro. En relación a las instituciones y profesionales entrevistados, la selección ha seguido también una pauta estratégica - agentes clave - y otra aleatoria a partir de la búsqueda de proyectos y el efecto "bola de nieve".

En total se ha entrevistado a 21 AMPA, 7 dinamizadoras en su mayoría contratadas por la misma $\mathrm{FaPaC}, 5$ directoras de centro y 9 representantes institucionales consorcios, ayuntamientos, servicios educativos del departamento de la Generalitat y entidades socioeducativas -. Cabe destacar un grupo focal realizado con una coordinadora de AMPAS y una reunión con miembros de AMPAS y de asociaciones de inmigrantes.

Durante el desarrollo de la investigación se tuvo en cuenta el análisis de las políticas educativas que el departamento de Educación ha puesto en marcha durante los últimos años, especialmente los Planes Educativos de Entorno, el Plan de Lengua y Cohesión y el Plan de Autonomía de Centros, fruto de lo cual se hicieron reuniones específicas con los servicios del Departamento que los dirigían.

\section{Resultados}

\section{Composición y dinámica de las AMPA}

El aspecto de la composición que destaca claramente es la fuerte correlación entre las 8 AMPA en contextos con presencia alta de familias inmigradas y la debilidad en su estructura organizativa, cuyas juntas formadas tan solo por 1, 2 o 3 personas (al cargo de todas las funciones que se creen más inmediatas) contrasta con el resto de la 
muestra, donde las AMPA están formadas por un mínimo de 8 personas y diferentes comisiones de trabajo.

A pesar de que en alguna de estas asociaciones estas pocas personas tienen una larga trayectoria en la escuela y una experiencia que les permite llevar cabo la mayoría de las actividades, en el resto de casos las pequeñas juntas tienen muy poca experiencia, con lo cual las actividades y responsabilidades de dichas AMPA quedan reducidas de manera drástica y generan una relación de dependencia con las direcciones de los centros muy fuerte, llegando a convertirse, a veces, en una organización testimonial:

"...lo hacen todo desde dirección, nosotros solo ponemos el dinero..." AMPA Vic.

Ahora bien, esta dependencia debe situarse en un contexto más general donde habitualmente se perciben las relaciones de las distintas direcciones hacia las AMPA como interesadas o viciadas, en las que el centro actúa como supervisor filtrando aquellas actuaciones o propuestas que pueden ser vistas como una injerencia en el ámbito profesional de los maestros:

"la relación es de servidumbre, hay servicios que cuestan mucho gestionarse, que no son obligatorios pero que son muy necesarios... se intenta transferir aspectos burocráticos a las AMPA” Dinamizadora 1.

Los comentarios sobre la falta de participación e información, la inutilidad de los órganos formales o la falta de entendimiento, se enmarcan en la complicada relación entre familias y escuela, de las cuales el AMPA tampoco se escapa - de hecho, no es nada extraño que las AMPA tengan, a la vez, problemas de entendimiento con la dirección y con el resto de padres y madres que no forman parte de la asociación -.

Es justo señalar, por otro lado, el esfuerzo y la visión favorable de la mayoría de las direcciones de los centros con mucha inmigración, ya que es su propia actuación e insistencia la razón de la existencia de algunas asociaciones.

En relación a las actividades y responsabilidades que las AMPA asumen como propias, se observa una prevalencia muy fuerte hacia las tareas de prestación de servicios al centro escolar y a los padres, centrados exclusivamente en los alumnos. En relación a las iniciativas para mejorar la participación y la acogida de las familias inmigradas, la mayoría de las AMPA lo perciben como una tarea que no les corresponde:

“¿Que qué puede hacer el AMPA? Poca cosa... esto tendría que ser una tarea del centro, cambiar la imagen de la escuela en el barrio para empezar..." AMPA bcn-2.

“...al fin y al cabo, esta acogida no deja de ser un trabajo más impuesto a las AMPA que tendría que hacer la administración.” AMPA Tortosa. 
Sin embargo, como veremos, otras AMPA no solo han iniciado proyectos interesantes en este sentido, sino que reflexionan y se cuestionan abiertamente sobre la idoneidad que las AMPA solo presenten este tipo de servicios:

"Entendemos que el AMPA y la participación tienen que ser efectivas y atractivas y por eso queremos convertirnos en un AMPA de movilización" AMPA Salt.

Un último aspecto importante a destacar es la escasa capacidad para movilizar a las familias en todas las AMPA. En más de un $85 \%$ de los casos se reconoce una dicotomía muy fuerte entre la participación que el AMPA organiza para los alumnos y las actividades de formación de padres y madres o debates sobre políticas educativas. Así, detrás de un AMPA compuesta por una sola persona y otra por más de 20, se esconden problemas similares a la hora de alcanzar a los padres cuando surge algún problema y se necesita la colaboración de todas las familias más allá de las fiestas y las actividades puntuales.

\section{Construcción y problematización de las familias inmigradas}

De las 21 AMPA visitadas, 2 cuentan con la participación muy activa de personas inmigradas extracomunitarias en la junta de AMPA y otras 2 tienen representantes en la junta aunque su participación es más bien puntual. En el resto de los casos, no existe ninguna representación por parte de madres o padres inmigrantes en la junta de AMPA. Por lo tanto, en la mayoría de casos la construcción de las familias inmigradas parte de una visión basada en el hecho de que ninguna de estas familias participa activamente en el AMPA, poniéndose de manifiesto los factores que se creen impiden o explican esta realidad.

El discurso más común al hablar de la participación de las familias inmigradas en el AMPA es el que he denominado "poca 'cultura de AMPA' y dificultades derivadas" y se basa principalmente en: 1 . Poner énfasis en las dificultades estructurales - situación socioeconómica e idioma - que se cree impiden la participación; 2. Añadir también el relativo eufemismo sobre su "poca cultura de AMPA" como factor también clave - ya fuera por desconocimiento de la asociación y cómo participar o como sinónimo de desinterés -; 3. Trasladar el peso de la responsabilidad hacia las familias y no tanto hacia lo que puede o no puede hacer el AMPA y/o el centro; y 4. Poner énfasis a las dificultades derivadas que esta falta de participación ocasionaban al resto de padres y madres del centro.

"la participación de estas familias es muy complicada"; "normalmente las familias inmigradas tienen unas prioridades muy diferentes a las de aqui"; "...que vengan y que no te entiendan cuando hablas"; "no tienen cultura participativa"; "son relativamente 'cerradas'” Extracto de varias AMPA. 
Los discursos construidos a partir de la denuncia de la segregación escolar arrojan una ambigüedad interesante. Por un lado, parten del uso de un lenguaje progresista y de claras referencias a la justicia social y al deber de denunciar y luchar contra estos procesos, en claras alusiones a la imposibilidad de tejer una sociedad intercultural a partir de escuelas "gueto" que muchas veces no responde a la realidad del propio entorno del centro. Por otro lado, el enquistamiento de este discurso parece imposibilitar cualquier marco de acción por parte de las AMPA más allá de la denuncia institucional y, en algunos momentos, roza el conservadurismo al reducir el problema de las familias - y los alumnos - inmigradas a un tema de números y porcentajes que pone en tela de juicio la capacidad del centro para conseguir una calidad educativa para todos los alumnos:

“...no sé, no estoy interesado con lo que se puede o no se puede hacer con estos alumnos (...) la repartición es una cuestión de justicia social, de equidad.” AMPA Ripoll.

El tercer discurso, mucho más puntual, lo encontramos en las aproximaciones no problemáticas, homogeneizadoras que rehúsan hablar en términos específicos de los alumnos y las familias procedentes de la inmigración:

"Que cuantas familias inmigradas tenemos? No sé, aqui estamos todos muy bien (...) El AMPA ya funciona muy bien, no necesitamos a más gente..." AMPA Olot.

"Aquí no tenemos alumnos inmigrantes, son de 2 a generación y no pueden ser tratados como extranjeros. No hay ningún problema a ese respecto" Directora, ben -1 .

"A nosotros no nos importa la procedencia de los alumnos(...) para nosotros todos son niños (...) aunque es lógico que en los grupos bajos hayan más inmigrantes" Directora, Olot.

El último tipo de discurso se aproxima al primero, ya que pone mucho énfasis en las dificultades estructurales que dificultan la participación de estas familias pero, en cambio, elude responsabilizarlas o etiquetarlas como menos interesadas que las autóctonas y traslada el peso de la iniciativa a las AMPA, los centros y la administración. En algunos casos, este discurso surgía puntualmente y mezclado con el primer o segundo tipo de discursos que hemos presentado en este apartado, lo cual indica no solo el carácter no concluyente de la construcción desde las AMPA sino también la visión variable de estas familias en función de un tema específico:

“...las familias inmigradas tienen otras dinámicas y necesitan otros tipos de servicios ya que tienen más sentimiento y ayuda comunitaria.” AMPA Vic.

"Las AMPA nos miramos mucho al ombligo". Reunión Coordinadora AMPA. 
"...lo primero es hablar con la gente, invitarlas a un café, que sientan que hablas con ellas, sin prisas, por la mañana cuando dejan a los niños..." AMPA Girona.

\section{Los proyectos de AMPA para mejorar la participación}

Diez de las AMPA de la muestra cuentan con una experiencia o proyecto de participación general o específico para las familias inmigradas: parejas lingüísticas, cursos de catalán, salidas familiares, meriendas compartidas, talleres de madres, atención personalizada, dinamización, sensibilización...

Estos proyectos representan un tercer tipo de actuación, que se sitúa entre la pura prestación de servicios o su función como "lobby" familiar, apareciendo como un espacio privilegiado de intervención socioeducativa.

De estos 10 casos, 3 proyectos se iniciaron claramente desde fuera del AMPA - 2 por parte de la dirección, otro por parte del ayuntamiento -, 2 proyectos habían sido iniciados de manera conjunta, aunque con una mayor iniciativa de las direcciones y el resto, 5, fueron puestos en marcha de manera autónoma por parte de las AMPA, en respuesta a situaciones que consideraban susceptibles de mejora, especialmente lo relacionado con las relaciones sociales entre todas las familias. Es importante subrayar como la mayoría de estos proyectos se iniciaron o han acabado dirigiéndose a todas las familias, quienes se han sentido atraídas por unos espacios nuevos que se visualizan como útiles y atractivos.

En general, la valoración que se hace es muy positiva, especialmente en el inicio, cuando se pone en marcha el proyecto, que contrasta con el momento anterior, en el que no había una actuación de este tipo. Es frecuente, también, las dudas sobre cómo amplificar el proyecto y acabar de acercar las familias a las AMPA.

No siempre la existencia de estos proyectos responde a una estrategia integral y compartida por la escuela dirigida conscientemente a asumir las actuaciones que sean necesarias para favorecer una mejor vinculación de las familias y de los alumnos. De forma análoga, no siempre tras un AMPA que no realiza ningún proyecto hay una indiferencia sobre este tema o se piensa que no es competencia del AMPA el dedicarse a tales actuaciones: de los 10 proyectos estudiados, muchos no tienen una continuidad en el centro o no se han identificado otras medidas realmente prácticas para favorecer dicha participación y, por otro lado, en 3 de los centros visitados, pese a no poseer ningún proyecto de AMPA específico, sí que tienen o bien una disposición más abierta, o se ha adoptado una mayor flexibilidad o se ha continuado con la participación normalizada de madres inmigrantes en el AMPA.

Hay otras experiencias, en contraste, que no han acabado bien. Este es el caso del trabajo que una entidad, en colaboración con $\mathrm{FaPaC}$, quería impulsar en dos escuelas y que, pese a contar con el apoyo de la junta del AMPA, tuvo que suspenderse porque casi ninguna familia se apuntó al taller. En general, es preciso atender a la complejidad en el desarrollo de tales proyectos y las múltiples variables que intervienen: tipología 
del proyecto, relación con el equipo de maestros, experiencia del AMPA, apoyo de personas clave - asesor LIC, mediadora cultural -, tipología de familias inmigradas, etc.

\section{Capacidad de intervención educativa de las asociaciones}

Más allá de la proclama muchas veces repetida de que el AMPA siempre actúa a favor del centro y de los alumnos, el tema del éxito escolar o, de manera más general, la reflexión en torno a la capacidad de intervención educativa de las AMPA se presentó, en primer lugar, como un tema espinoso y esquivo, al que era difícil responder de una manera clara.

En segundo lugar, puede observarse como la totalidad de las AMPA entrevistadas desconocen el Plan de Acogida de Centro, las medidas de atención a la diversidad, la organización curricular general e incluso los propios proyectos de autonomía de los centros que disfrutan de éstos. En general hay mucho desconocimiento sobre las trayectorias posteriores del alumnado, especialmente del alumnado inmigrado, como muestran los contrastes entre una primaria donde "todo va muy bien" y el traspaso a secundaria:

"La mama de x no se enteró de que su hijo faltaba a clase y que no hacía nada (...) tenía muchos problemas para comunicarse con el instituto" AMPA Igualada.

"Aqui tenemos una organización de escuela y un equipo de maestros excelente, tratan muy bien a los niños (...) el problema es en secundaria, alli trabajan muy diferente" Directora, bcn -4 .

Las cuestiones de éxito escolar parecerían recaer exclusivamente en los propios padres de los alumnos, lo cual estaría en contradicción con la importancia central otorgada a la calidad de enseñanza del centro y que se mostraba en la existencia de unos claros límites a la intervención del AMPA - y la familia - en las cuestiones educativas, que reforzaban la centralidad del "profesionalismo" educativo representado por las maestras y la escuela:

"El AMPA es un reflejo de la sociedad y la educación tiene que estar en manos de los profesionales; ahora bien, asumiendo que quién tira de la cuerda son los padres: si los padres no están detrás, el fracaso está garantizado" AMPA ben -3.

Lo que deja bastante claro cuáles son los límites en la intervención educativa de las AMPA: éstas no tendrían que intervenir en espacio y aspectos puramente escolares ni debería distanciarse de la dinámica de la escuela como, de hecho, se pone de manifiesto en todas las entrevistas. Por lo tanto, parecería prevalecer la función instrumental y "de servicios" de las AMPA que funcionaría como una garantía de 
recursos extra para la escuela y mejorar la imagen a partir de los servicios que ésta presta. Sin embargo, un análisis más detenido de ciertos detalles, así como algunos comentarios sobre los servicios que realizan, deja entrever cómo desde las asociaciones se percibe la prestación de estos servicios como una cierta actuación educativa:

\section{“...los servicios que prestamos se pueden considerar como educativos ya que compensan deficiencias educativas detectadas" AMPA bcn -3.}

En esta misma línea se ubican aquellas actuaciones mucho más informales que tienen un impacto educativo importante, como es el caso de un AMPA donde, a partir de los contactos de algunos miembros con la administración local, permitían ayudar económicamente a las familias más necesitadas o buscar más recursos para la escuela. O también otra asociación donde se asesoraba informalmente a las familias con más dificultades sobre cuestiones de papeleo y procedimientos burocráticos.

\section{La visión institucional y los proyectos de intervención con las AMPA}

A raíz de los resultados de las visitas a las direcciones de los departamentos de educación responsables de impulsar las nuevas políticas educativas basadas en una perspectiva sociofamiliar, se ha identificado el uso de un doble discurso basado en poner énfasis en el trabajo educativo más social y comunitario pero que no está implementando actuaciones concretas ni amplificando las existentes, ni acompañándolas ni evaluándolas.

Los institutos municipales de educación visitados así como varias entidades de renombre también hacían gala de este doble discurso, mostrando mucho interés por los potenciales de los proyectos de AMPA pero obviando la existencia de proyectos concretos.

En el otro extremo tenemos los casos de un Instituto Municipal, que había intentado desarrollar trabajos concretos con las AMPA o de una entidad socioeducativa que contaba con una amplia experiencia de trabajo conjunto con los centros educativos y las familias, para quienes el trabajo con las AMPA debería "capacitarlas a partir de analizar qué perfiles de participación tienen y necesitan, saber cómo trabajan y que grietas podemos encontrar para convocar a las madres y al resto del colectivo de padres".

Sobre los Planes Educativos de Entorno, hallamos una opinión crítica por parte de las AMPA, donde se desprende el alcance limitado de lo que ha sido la punta de lanza de la intervención de la administración para mejorar la cohesión social y los resultados educativos de barrios marginales, populares y/o con altos índices de población extranjera, el modelo de "catálogo de servicios" en que se había convertido y, no en pocos casos, la falta de participación, transparencia y competencia indirecta con funciones que ya acometían las AMPA. 
Por último, tenemos la relativa incidencia de los proyectos de dinamización de AMPA que no solo impulsa $\mathrm{FaPaC}$, sino también otros municipios e incluso uno de los centros educativos visitados. En general, se percibe la falta de coordinación y de una visión clara de objetivos y modelo de asociacionismo a impulsar, lo cual deja a la mayoría de profesionales bajo su propio criterio a la hora de encarar las muchas y diversas situaciones con que se encuentran, a lo que se suma una falta patente de recursos para atender a las AMPA en condiciones, donde unas 20 dinamizadoras a tiempo parcial deben atender el total de las 1900 AMPA federadas.

\section{Discusión: ¿un lugar para las asociaciones?}

\section{¿AMPA como asociaciones intermediarias?}

Atendiendo a la formulación que López et al. (2005) hacen de las organizaciones intermediarias, es indudable que el binomio AMPA-FaPaC se situaría en la línea de las "entidades sin ánimo de lucro que operan entre las familias y los recursos para mejorar la participación" (2005:79). Sin embargo, esta posición estratégica que juegan las asociaciones de padres y madres se diluye debido a su dedicación casi exclusiva a la prestación de servicios, debilitando las posibilidades de fortalecer la participación y colaboración entre padres y escuela.

En primer lugar, las actividades de formación, debate y mejora de la comunicación con el centro son objetivos explícitos de FaPaC y de no pocas AMPA, así como su papel de interlocutora de la opinión de las familias ante la escuela. Ahora bien, el carácter puntual de dichos consejos y charlas y el relativo éxito de muchos de los talleres de formación que se llevan a cabo, demuestran una competencia débil en este sentido.

En segundo lugar, no existe un objetivo explícito para desarrollar un sentido de liderazgo entre los miembros de las AMPA, ni de establecer espacios de colaboración y formación recíprocas entre padres y profesionales que favorezca un acercamiento de la cultura escolar a la familiar.

En tercer lugar, en la mayoría de los casos se percibe un cierto hermetismo de las asociaciones por los asuntos concretos e inmediatos de su propia escuela y una escasa y puntual colaboración con otras entidades del contexto escolar. Además, la relación de $\mathrm{FaPaC}$ con las AMPA dista de ser cercana y continua, por lo que podríamos afirmar, siguiendo a Garreta (2008a), que estamos hablando de dos movimientos paralelos pero independientes y relativamente distanciados, lo cual limita las posibilidades de las AMPA para acceder a recursos técnicos o para adquirir un discurso más politizado o reflexivo sobe el modelo de AMPA a impulsar o sobre su papel como agente de la sociedad civil en la defensa de los derechos sociales (Garreta, 2008a).

La falta de sistematización de las actuaciones impulsadas, especialmente desde las entidades externas - FaPAC, Departamento, Ayuntamientos -, su distanciamiento con el día a día de la escuela y la falta de evaluación de resultados dibujan una situación que está lejos todavía de poder provocar una mejora de la participación por parte de las 
familias inmigradas o de acercar el espacio del AMPA al espacio académico, imposibilitando su verdadera actuación como asociaciones intermediarias.

\section{¿Son los proyectos de AMPA una herramienta para alterar las dinámicas de participación?}

Los proyectos de AMPA iniciados para mejorar la relación entre todos los padres o las competencias lingüísticas y culturales son el ejemplo más claro sobre cómo las AMPA podrían ejercer un papel más allá de la prestación de servicios o de ser la correa de transmisión de ciertas demandas de las familias del centro.

Tanto los talleres, como los cursos, la creación de parejas lingüísticas o, sencillamente, el compartir espacios - meriendas o salidas en familia - generan una posibilidad de mejora del capital social de las familias, de establecer nuevas relaciones fuera del colectivo como también capacita funciones de liderazgo entre los miembros del AMPA que impulsan tales proyectos.

Pero también es cierto que, a excepción de 2 de estos proyectos, el resto no son sino actuaciones puntuales que difícilmente podrían desembocar en un cambio en las dinámicas de participación y presencia de familias inmigradas en las AMPA o en la escuela, como de hecho todos ellos reportan, quedando también lejos de las actuaciones que Abrams \& Gibbs (2002) muestran como necesarias para la alteración de los procesos de exclusión o marginación de las familias inmigradas en la escuela. El hecho de no existir una avaluación inicial, ni un trabajo por objetivos ni una evaluación rigurosa final limita el alcance de estos proyectos, así como sus consecuencias para la presente discusión, como también señalan en el mismo sentido múltiples estudios (López et al., 2005; Corter \& Pelletier, 2005; Desforges \& Abouchaar, 2003).

De manera general, los proyectos parten de un no cuestionamiento de las expectativas "ocultas" (Crozier \& Davies, 2007) que caracterizan la estructura de participación de las familias en la escuela y la creación de los espacios ocupados por uno y otros en base a unas tradiciones y pautas culturales específicas (Theodorou, 2007), planeando en el fondo siempre el carácter asistencial y bienintencionado en tales actuaciones. Esta dificultad para articular la actuación del AMPA con los procesos escolares hace que sobresalgan las actuaciones y justificaciones de tipo más discursivo, ligada al reconocimiento y celebración de la diversidad cultural que dificulta su capacidad de vehicular actuaciones que incidan en cuestiones más estructurales (Hart, 2008).

Como contrapunto, señalar que la existencia de discursos críticos que reconocen la desigualdad estructural del nuestro sistema social y el lugar subalterno que muchas familias inmigradas ocupan, junto a algunos proyectos de AMPA más diversificados y continuos, sí que podrían ser la base para un cambio en las dinámicas de participación de las familias inmigradas en el centro, al menos potencialmente. 
¿Son las AMPA un espacio demasiado formalizado para la participación de los grupos más desfavorecidos?

Pese a la existencia de barreras que bien podrían atribuirse tanto a un grado de formalismo en su funcionamiento como a la existencia de discursos que favorecen la exclusión de estos grupos, es la poca flexibilidad y el escaso cuestionamiento de los modelos de participación que se espera de las familias por parte de toda la comunidad educativa, por un lado, así como la errática identidad de unas asociaciones cuyo espacio está siendo cada vez más desplazado y cuestionado por las perspectivas más individualistas de relación familia y escuela basadas en el modelo consumista (Bolívar, 2006; Dias, 2005, Subirats et al., 2003) por otro, lo que nos ayudaría a situar la verdadera problemática a la hora de poder participar en y de las AMPA.

Si en muchos de los centros segregados el AMPA solo existe bajo la participación de una o dos personas que, en conveniencia con la dirección, son capaces de construir la plataforma básica que justifica su existencia - atraer recursos, organizar servicios ¿qué necesidad hay para implicar a más familias? Además, en los casos donde esta asociación no existe, la experiencia en la creación de AMPA en aquellos contextos particularmente difíciles nos muestra como, en efecto, los requerimientos formales son una dificultad añadida pero accesoria - gracias, en buena parte, al asesoramiento de las dinamizadoras. Lo cual nos podría llevar a interrogarnos si los servicios que presta son adecuados a las necesidades de buena parte de las familias inmigradas.

\section{¿Las instituciones apuestan por un espacio real de fortalecimiento de las redes sociales de las familias?}

Si bien en Cataluña no ha habido una reforma legislativa clara que afiance de manera directa la participación de los padres en la escuela como los casos descritos por Dias (2005) o Kristoffersson (2007), en Portugal o Dinamarca, respectivamente, el carácter e impacto que finalmente están teniendo estos planes e iniciativas, más allá de las bondades propositivas y metodológicas que algunos de manera un tanto ingenua no se cansan de proclamar sin evaluación alguna (Martí, 2008; Besalú \& Vila, 2005), es la de reforzar un doble proceso. Mientras que las medidas compensatorias están teniendo un alcance limitado, las escuelas y las familias, de manera efectiva, están creando maneras ad hoc para responder a la diversidad cultural a través de la segregación interescolar o intraescolar - grupos de nivel -, esta última gran desconocida para muchos padres y defendida por el sentido común cuando se planteó en las entrevistas.

La casi inexistencia de proyectos que cuenten con las AMPA, a diferencia de su uso en la externalización de servicios educativos básicos - a través de las cuantiosas subvenciones otorgadas por el Departamento -, deja una imagen bien definida y monolítica que de las AMPA y las familias tienen la mayoría de poderes públicos de facto, no en el discurso: las unas como prestadoras de servicios, las otras como meras consumidoras. 


\section{Conclusión: una posibilidad aún por construir (y analizar).}

Los resultados expuestos y la discusión presentada apuntan a la difícil creación, a través de las AMPA, de esos espacios intermedios que sirvan para reformular las narrativas hegemónicas, plantear cambios organizativos que a su vez alteren dinámicas de exclusión o liderar los procesos de formación y empoderamiento de las familias que López et al. (2005) apuntan.

De esta forma, el capital social que se inscribe en los procesos de diferenciación, producción y reproducción de los grupos sociales parecería quedar reducida a las decisiones individuales, quedándose las posibilidades de colectivización e intercambio entre los distintos grupos diluidas entre la creciente segregación y diferenciación escolar o la gestión empresarial de los centros.

Lejos de representar, simplemente, otro espacio más de exclusión social, el lugar subordinado de las familias inmigrantes en la escuela y las AMPA no hace sino manifestar con mayor fuerza aún la existencia previa de unas tendencias no resueltas que afectaban ya a muchos grupos sociales no hegemónicos y que ahora podrían naturalizar los procesos de exclusión a través de una democratización de la culpa hacia los grupos más desfavorables (Dahlstedt, 2009).

Pese a todo, es importante señalar el alcance limitado y provisional de la presente investigación. Tres elementos nos obligan a ser prudentes en este punto. En primer lugar, la existencia de los proyectos de AMPA estudiados, aun incipientes y puntuales, justifican esta perspectiva propositiva como asociaciones intermediarias. En segundo lugar, el hermetismo y localismo de muchos centros, así como su dispersión, podrían ocultar la existencia de otras intervenciones más exitosas que serían necesarias avaluar. $\mathrm{Y}$ en tercer lugar, la falta de proyectos integrales y bien diseñados que nos hubieran permitido una verdadera contrastación con la propuesta de asociaciones intermediarias recogida de otros contextos, nos impiden ser concluyentes en relación con las posibilidades de las actuaciones de las AMPA y sus federaciones territoriales.

La generación y aprovechamiento de capital social colectivo a través de las AMPA y su papel como agente para revertir dinámicas de exclusión socioeducativa dependería, también, del contexto de movilización social más amplio y de su ubicación dentro de un proyecto político colectivo en una época cada vez menos proclive a ello.

\section{Referencias bibliográficas}

ABRAMS, L. Y GIBBS, J. (2002). Disrupting the logic of home-school relations: parent involvement strategies and practices of inclusion and exclusion. Urban Education, 37, 3: 384-407.

BESALÚ, X. Y VILA, I. (2005). Consolidar la cohesió social, l'educació intercultural i la llengua catalana. Caixa d'eines, 1, 6-30. Consultado el 10/6/2010 en: http://www.xtec.es/lic/intro/caixa.htm\#1 
BOETHEL, M. (2003). Diversity: school, family y communitiy connections. Anual synthesis 2003. SEDL: Austin, Texas.

BOLIVAR, A. (2006). Familia y escuela: dos mundos llamados a trabajar en común. Revista de educación, 339,119-146.

BROWN, P. (1990). The 'third wave': education and the ideology of parentocracy. British Journal of Sociology of Education, 11(1), 65-85.

CARRASCO, S., PÀMIES, J. Y BERTRAN, M. (2009) "Familias inmigrantes y escuela: desencuentros, estrategias y capital social". Revista Complutense de Educación, Vol.20 Núm.1.

CARRASCO, S., PÀMIES, J., PONFERRADA, M., BALLESTÍN, B. Y BERTRAN, M. (2010) Segregación escolar e inmigración en Cataluña: Aproximaciones etnográficas. En: F. J. García Castaño y S. Carrasco (eds.) Investigaciones en Inmigración y Educación en España. Homenaje a Eduardo Terrén Lalana, Madrid: CIDE-Ministerio de Educación.

CORTER, C. Y PELLETIER, (2005) Parent and Community Involvement in schools: policy panacea or pandemic?, International Handbook of Educational Policy, 295327. Springer: Great Britain.

CROZIER, G. (2001) Excluded Parents: the deracialisation of parental involvement. Race Ethnicityand Education, 4: 4, 329- 34.

CROZIER, G. Y DAVIES, J. (2007). Hard to reach parents of hard to reach schools? A discussion of home-school relations, with particular reference to Bangladeshi and Pakistani parents. British Educational Research Journal, 33, 3: 295-313.

DAHLSTEDT, M. (2009). Parental governmentality: involving 'immigrant parents' in Swedish schools. British Journal of Sociology of Education, 30(2), 193-205.

DE CARVALHO, M.P. (2001). Rethinking familiy-school relationships: A critique of parental involvement in schooling. Mahwah NJ: Lawrence Erlbaum.

DEPARTAMENT D’EDUCACIÓ (2004). Pla de Llengua i Cohesió Social. Página consultada el 9/07/2010:

http://www20.gencat.cat/docs/Educacio/Home/Arees_actuacio/innovacio_educativa/P1 a\%20de\%20convivencia/pla_lic_nov_09.pdf

DEPARTAMENT D’EDUCACIÓ (2005). Els Plans Educatius d'Entorn. Página consultada el 9/07/2010:

http://www20.gencat.cat/docs/Educacio/Home/Arees_actuacio/innovacio_educativa/Pl ans\%20educatius\%20entorn/doc_marc_pee_cat.pdf

DESFORGES, C. Y ABOUCHAAR, A. (2003). The Impact of Parental Involvement, Parental Support and Family Education on Pupil Achievements and Adjustement: A Literature Review. Department for Education and Skills, Research Report, 433.

DIAS, M. (2005). Market trends and the dilemmas of parental participation in schools. Aula Abierta, 85, 185-204. 
DRIESSEN, G., SMIT, F. Y SLEEGERS, P. (2004) Parental Involvement and Educational Achievement. British Educational Research Journal. Vol. 31, 4, 509532

DWYER, C. Y MODDOD, T. (2006). Ehnicity as social capital? Explaining the differential educational achievements of young British Pakistani men and women. Paper presented at the "Ethnicity, Mobility and Society" Leverhulme Programme Conference at University of Bristol, 16-17 March.

EURYDICE (1997). El Papel de los padres en los sistemas educativos de la Unión Europea. Bruselas: Unidad Europea de EURYDICE.

EPSTEIN, J.L. et al. (2002). School, family and commnunity partnerships: your handbook for action. California: Corwing Press.

FEITO, R. (1992). Llamando a las puertas de la escuela: la participación de los padres en el control y gestión de la enseñanza. Trabajo de Tesis. No publicado.

GARRETA, J. (2008a). La Participación de las familias en la escuela pública: las asociaciones de madres y padres del alumnado. Madrid: CIDE y CEAPA.

(2008b) Escuela, Familia de origen Inmigrante y participación. Revista de Educación, n³45: 133-155.

GITLIN, A. et al. (2003). The Production of Margin and Center: WelcomingUnwelcoming of Immigrant Students. American Educational Research Journal, 40, 1:91-122.

GOMOLLA, M. (2006). Tackling underachievement of learners from ethnic minorities: a comparison of recent policies of school improvement in Germany, England and Switzerland. Current Issues in Comparative Education, 9, 1: 46-59.

GRAHAME, P.; GRAHAME, K. (2001) Official Knowledge and the Relations of Ruling: explorations in institutional Ethnography. The Journal for Pedagogy, Pluralism, \& Practice. Página consultada en 12/7/2010: http://www.lesley.edu/journals/jppp/5/grahame.html

HART J. (2008). "Children's Participation and International Development: Attending to the Political", International Journal of Children's Rights, 16: 407-418.

KRISTOFFERSSON, M. (2007). The paradox of parental influence in Danish schools. A Swedish Perspective. International Journal about Parents in Education, v.1, 0, 124-131.

LAREAU, A. (1987). Social-Class Diferences in family-school relationships: the importance of Cultural Capital. Sociology of Education, 60: 73-85.

LAREAU, A. Y HORVAT, E.M. (1999) Moments of social inclusion and exclusion: race, class and cultural capital in family school relationships. Sociology of Education, 72, 37-53. 
LAVAL, C. (2005). Per què l'escola no és una empresa?. Descargado el 20/7/2009 en: http://www.uoc.edu/dt/cat/laval0505.pdf

LÓPEZ M. E., KREDIER, H. Y COFFMAN, J. (2005). Intermediary organizations as capacity builders in family educational involvement. Urban Education, 40, 1, 78105.

MARTÍNEZ CERÓN, G. (2004). "La participación de los padres y las madres. Eje del modelo educativo de CEAPA". Cuadernos de pedagogía, 333, 46-49.

MARTÍNEZ-GONZÁLEZ, R.A. \& PÉREZ-HERRERO, M.H. (2006). "Propuestas metodológicas para una educación de calidad a través de las relaciones entre centros docentes, familias y entidades comunitarias". Cultura y Educación, 18(3/4), 231246.

MARTÍ, Q. (2008). El Pla LIC i la seva concreció als centres educatius. Guix, 341, 913.

MONKMAN, K., RONALD, M. Y THÉRAMÈNE, F. (2005) Social and cultural capital in an urban latino school community. Urban Education, 40(1), 4-33.

PAÑELLAS, M. Y ALGUACIL, M. (2008). "La participació de les famílies als IES" Informe de Investigación. No publicado.

POOMERANTZ, E.; MOORMAN, E; LITWACK, S. (2007). The How, Whom and Why of Parents' Involvement in Children's Academic Lives: More is Not Always Better. Review of Educational Research, 77, 3, 373-410.

STANTON-SALAZAR, R. (1997). A Social Capital Framework for Understanding the Socialization of Racial Minority Children and Youths. Harvard Educational Review, 67, 1: 1-40.

SANTOS GUERRA, M.A. (1997). El crisol de la participación. Investigación sobre la participación en los Consejos Escolares de Centro. Madrid. Escuela Española.

SARRAMONA, J. (2001). Recerca sobre la participació dels pares al sistema educatiu. Consejo Escolar de Cataluña.

SYMEOU, L. (2005). Past and present in the notion of school-family collaboration. Aula Abierta, 85, 165-184.

SUBIRATS, J. et al. (2003). Quina participació per a quina societat? Publicació interna de $\mathrm{FaPaC}$.

TAPIA, J. (2002). Reexamining Anthropology and Education: Bringing Economics back to Culture. Urban Education, 37, 3: 365-383.

THEODOROU, E. (2007). Reading between the lines: exploring the assumptions and implications of parental involvement. International Journal about Parents in Education, v.1, 0, 90-96. 
TORRES SÁNCHEZ, M. (2004). El capital social en las asociaciones de madres y padres: formación, desarrollo e institucionalización. Granada, editorial Universidad de Granada (Tesis Doctoral en CD-ROM).

TAYLOR, M. \& PERCY-SMITH, B. (2008). "Children's Participation: Learning from and for Community Development", International Journal of Children's Rights, 16, 379-394.

VAN ZANTEN, A. (1997). Schooling Immigrants in France in the 1990's: Success or Failure of the Republican Model of Integration? Anthropology y Education Quarterly, 28, 3: 351-374.

WARREN, S. (2007). "Migration, race and education: evidence-based policy or instituional racism?". Race, Ethnicity and Education, 10, 4:367-385.

\section{Correspondencia con el autor}

Alejandro PANIAGUA RODRIGUEZ

Departamento de Antropología Social y Cultural. Universidad Autónoma de Barcelona Facultat de Lletres. Edifici B.

08193 Cerdanyola del Vallès

(Barcelona, España)

Correo-e: alejandro.paniagua@uab.cat 